\title{
TMJ and posture, yes it's all connected: the chain theory of body linkage
}

\section{Editorial}

Posture is a complex multifactorial problem that can be affected by different systems including Neurological, visual, musculoskeletal, and somatosensory. Postural adjustments are the result of a complex system of mechanisms that are controlled by multisensory inputs (visual, vestibular, and somatosensory) integrated in the CNS. The stomatognathic system (especially the TMJ and occlusion) also plays an important role in postural control. If you have a malocclusion, the muscle tension and activity will also be unbalanced. When those muscles are over-contracted, it impacts and 'pulls' the muscles that hold up your head creating a forward head posture. This slouching action is mimicked down your body, pulling and stressing your face, neck, shoulder and back muscles which can result in having one shoulder or hip higher than the other.

TMD is multifactorial and sometimes it's hard to realize the reason behind it. One of the unnoticed reasons of the TMD is the bad posture. The chain theory of body linkage explains the relation between the bad posture and TMD. The chain consisted of interconnected three rings, upper, middle and lower ring. The upper ring consisted of the muscle of the TMJ, head and spine. The middle ring consisted of the muscle of the back and vertebra. While the lower ring consisted of the muscle of the foot, ankle, and legs. For normal daily function, each of these rings must work in a co-ordinated and synchronization mode with the other rings.

The biomechanical complexity of the posture drives from the functional integration of the chain of rings of the body segments. If one ring of the chain is stressed, it affects the other two rings in the chain and pain can be involved the whole body from toes and heal up through our jaw. This is resulting from functional adaption and realignment of the chain of the rings of the body linkage. As a result, deviation of the lower ring of the chain will not only interfere with the posture organization but also it will have an impact on the TMJ and the group of stomatognathic system which belong to the upper ring of the chain and vice-versa.

The physiology involved in the integration between the rings of the chain explained by many biomechanical theories which based on that, the change in the tissue tension-compression in one ring of the muscles chain will lead to changes of the tissue tension-compression leading to bad posture. Some other theories based on the electro myographic activity of the muscles in one ring of the chain which associated with elongation of shorting of the other rings within the muscle chain.

Patients with TMD reported to be associated with decrease of the posterior facial height with backward and clock wise rotation of the mandible. This features influence the Craniocervical posture. Patients with TMD and cervical spine problem (upper ring) associated with head moved forward. Normally, the head sit directly over the neck and shoulders, change in the posture with forward head posture lead
Volume 2 Issue 6 - 2015

\author{
Ayman Hegab \\ Department of Oral \& Maxillofacial Surgery,Al-Azhar University, \\ Egypt \\ Correspondence: Ayman Hegab, Clinical Associate Professor \\ of Oral \& Maxillofacial Surgery,Al-Azhar University, Egypt, Tel \\ 97433310124, Fax 0020100198III2, \\ Email hegab@gmail.com, prof.aymanhegab@yahoo.com
}

Received: July 26, 2015 | Published: August 28, 2015

to a sequence of events. Forward head movement cause strain on the muscle of the neck and shoulders and upper back muscle. This cause decrease of the blood supply of the muscle with muscle fatigue and pain. This movement led to shifting the center of the gravity. As a result, the upper body and back (middle ring) try to compensate by drafting backward. While the hip and lower body (lower ring) moving forward. So, the TMD and neck problem can result in middle and lower back problem, due to loss of balance between the rings of the body chain.

High heels are very popular with many women and can be one of the reasons of increasing the incidence of the TMD within the women group of patients. The higher the heel, the more potential damage you can cause to the ankle and foot, by shortening the Achilles tendon that runs behind the heel to the bottom of the foot. This lead to tighten and shortening of the tendon resulting in heel pain. Also, its change the angle of the heel to the toes thereby changes our center of gravity; the Calf muscles contract and adjust to the angle of the high heel and walking with high heel making excessive force within the knee (lower ring). The high heels push the center of the body forward taking the hip and spine put of the normal alignment (middle ring). All of the previously mentioned changes lead to posture alteration which affects the Craniocervical and TMJ muscles (upper ring).

This changes the alignment of the hips and spine, (which include our head and neck), predisposing us to back and leg pain or pain anywhere up the chain of linkage. Wearing of sport comfortable shoes can relief the muscle strain in the lower body (lower ring) with subsequent improvement of the bad posture. The result of improving the bad posture will be reelected on the TMD and the neck (upper ring). Gordon ${ }^{1}$ discussed the functional and anatomical relationship between the jaw, head, cervical spine and pelvic complex in one study. Fink et al., ${ }^{2}$ had twenty subjects undergo a procedure to create an artificial dental occlusal interference. They found a statistically significant occurrence of hypo mobile functional abnormalities following the dental modification, and they concluded that, Body posture was more 
stable when subjects bit down in centric occlusion. Changes in body posture affected occlusal force distribution. Altering body posture by changing leg length shifted the occlusal force distribution to the same side that had a heel lift. The use of occlusal splint utilized in the treatment of the TMD should be incorporated in the multidisciplinary teamwork for treatment of the posture alternation. The occlusal splint will help in adjustment of the head posture and this will be reflected on the total body posture.

Factors affecting the posture including TMD, high heel (as mentioned before). Moreover, children with heavy backpack to carry the school books, long time using the computer with positioning of the screen at lower level lead to forward of the head. Long time watching and playing the videogames especially in children not only lead to bad posture but also lead to body adaptation to the new bad posture. Points to remind:

a) Remember to check the TMJ and cervical area. Adjusting the bite may be needed as part of an integrated postural improvement program.

b) Wear sport comfortable shoes. No high heel shoes.

c) Sit up in straight position (try to align the head over the neck and shoulders)

d) Support your back with a back pillow during sitting for long time. e) decrease the weight of the backpack (maximum backpack should not more than $15 \%$ off the child weight).

f) The pillow should be roughly as thick as the distance between your ear and your shoulder.

g) During back-sleeping, the back of your head should be in contact with your mattress. A pillow that holds the back of your head above your mattress reinforces forward head posture. A pillow that held your head in a forward position all night will lead to walking and standing with that same forward head posture. Therefore your pillow should provide support under your neck rather than under your head. The neck support can be provided by $U$ shaped pillows, a rolled up towel, or a pillow with a hole in the middle.

h) Improve your resting posture is by holding yourself as tall as possible.

\section{References}

1. Gordon C. Diagnosis and co-management of temporomandibular joint disorders: a case study. The British Journal of Chiropractic. 1999;3(2):40 43.

2. Fink M, Wahling K, Stiesch-Scholz M, et al. The functional relationship between the craniomandibular system, cervical spine, and the sacroiliac joint: a preliminary investigation. Cranio. 2003;21(3):202-208. 